\title{
Comparison of dose requirement of propofol with and without bispectral monitoring in patients undergoing spinal epidural anaesthesia: An institutional based study
}

\author{
Ratan Pal Singh', Megha Arora ${ }^{2, *}$ \\ ${ }^{\mathbf{1}}$ Associate Professor, ${ }^{\mathbf{A}}$ Assistant Professor, Dept. of Anaesthesia, Rajshree Medical Research Institute \& Hospital, Bareilly, Uttar \\ Pradesh, India
}

*Corresponding Author: Megha Arora

Email: dr.meghaarora@gmail.com

Accepted: $29^{\text {th }}$ June, 2018

\begin{abstract}
Introduction: Sedative protocols are a topic of current research, especially for establishment of extensive monitoring systems for sedative agents. Bispectral index (BIS) is one of the main indicators for measuring the anaesthesia depth. Hence; present study was planned to comparatively assess the dose requirement of propofol with and without bispectral monitoring in patients scheduled to undergo spinal epidural anaesthesia.

Materials and Methods: We planned the present research to comparatively assess the dose requirement of propofol with and without bispectral monitoring in subjects scheduled to undergo spinal epidural anaesthesia. For the present study, we evaluated a total of 60 patients and broadly divided them into group 1 and group 2, based on the absence and presence of BIS monitoring respectively. We maintained and compiled the complete demographic, clinical and anaesthetic data of all the patients and analyzed the results by SPSS software.

Results: While comparing the average time needed for reaching the adequate level of sedation in between the two study groups, significant results were obtained. We also obtained significant results while comparing the average recovery time in between subjects of both the study groups.

Conclusion: For assessing the level of sedation in regional anaesthesia cases, BIS monitoring should be done.
\end{abstract}

Keywords: Bispectral monitoring, Propofol, Spinal anaesthesia.

\section{Introduction}

Anaesthesia depicts the perfect coordination between anesthetics agent need and the state of arousal of the subject undergoing anaesthesia procedure. If any inadequacy exists in relation to the dose of the anaesthesia, there is increase in prevalence of awareness. Also hemodynamic instability, delayed recovery, along with incidence of other complications occur with generous administration of drugs. ${ }^{1,2}$

In the recent era, there has been extensive investigation on the sedative protocol because of advancement sin the monitoring systems of sedative drugs. Significant tolerance and speedy recovery has been found to be occur when intermittent propofol is used for sedation. ${ }^{3}$

Propofol is a preferred agent because of its capacity to preserve constant plasma concentration with non-stop infusion. Shorter induction time along with rapid redistribution is some of the other advantages offered by propofol. Required propofol dose is reduced by addition of opioids to it. ${ }^{4-6}$ Bispectral index (BIS) is one of the commonly employed indicator for assessing the death of anaesthesia. Patients' electroencephalography (EEG) and electromyography (EMG) statistics are assessed by it. Data from the past shows positive association between propofol drug concentration, and BIS level. ${ }^{7-9}$

Under the light of above mentioned data, present study was planned to assess and compare the dose requirement of propofol with and without bispectral monitoring in patients undergoing spinal epidural anaesthesia.

\section{Materials and Methods}

The present study was commenced in the Anaesthesia department of Rajshree Medical Research Institute \& Hospital, Bareilly, Uttar Pradesh (India) and it included assessment of the dose requirement of propofol with and without bispectral monitoring in patients undergoing spinal epidural anaesthesia. Enrolment of 60 patients was done in the present study who underwent elective gynecological surgical procedure. This was followed by random division of patients into two study groups with 30 patients in each group as follows:

1. Group 1: Patients who received propofol infusion without BIS monitoring,

2. Group 2: Patients who received propofol infusion with BIS monitoring.

\section{Exclusion Criteria}

1. Patients with presence of any co-morbid condition.

2. Patients with positive history of drug allergic reactions.

3. Patients with presence of psychiatric disorder.

4. Patients with presence of neurological disorder.

Recording of complete clinical and biochemical details of all the subjects in the operating room was done. Complete data relation to the time of infusion, recovery and postoperative compilations were maintained. SPSS software was used for assessing the 
results with univariate regression curve and Mann Whitney $\mathrm{U}$ test for assessing the significance level.

\section{Results}

Broad division of 60 subjects was done in the present study into two study groups. Mean age of the subjects of group 1 was 40.5 years. Mean age of the subjects of the group 2 was 42.1 years. Average time required for reaching the sedation satisfaction level was 18.5 minutes in group 1 and 7.3 minutes in group 2 . The results of this comparison were found to be statistically significant.10.45 minutes was the mean recovery time observed in patients of group 2 while 5.42 minutes was the mean recovery time observed in patients of group 1, the results of which were found to be statistically significant. We didn't observe any significant difference while comparing the frequency of complications in between the two study groups.

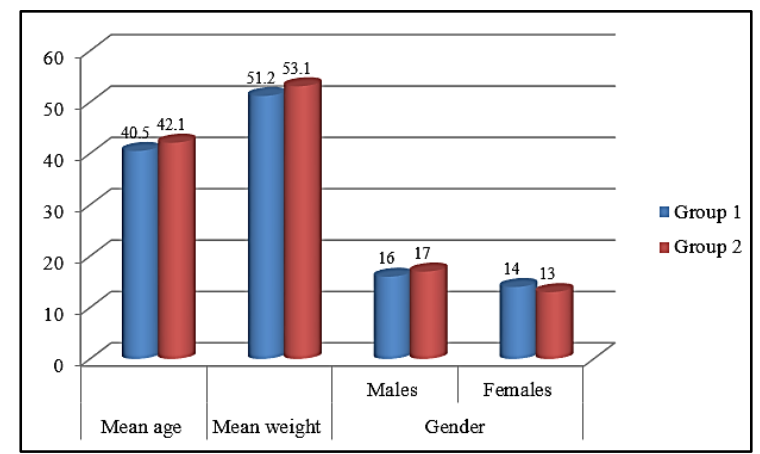

Fig. 1: Description of patients

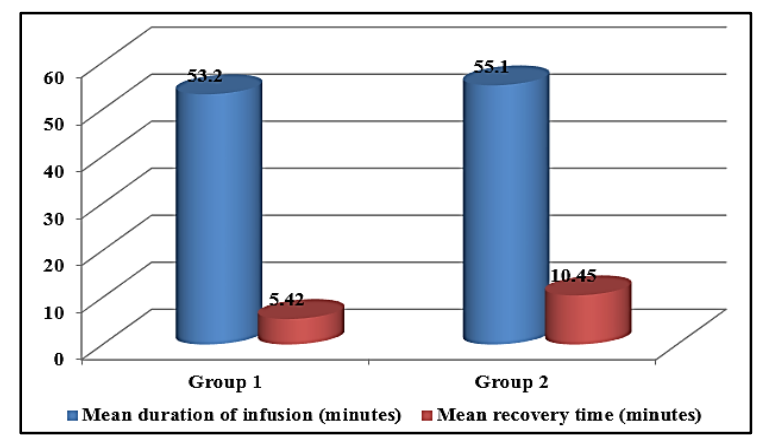

Fig. 2: Mean duration of infusion and recovery time among subjects of both the study groups

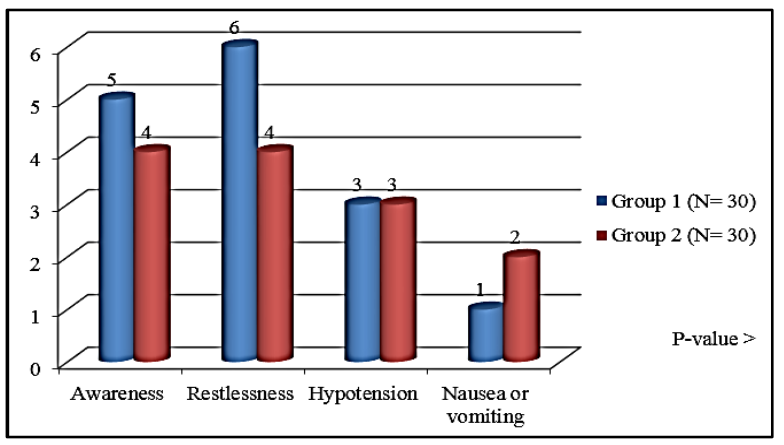

Fig. 3: Complications
Table 1: Comparison to time duration in between the two study groups

\begin{tabular}{|l|c|c|c|}
\hline \multicolumn{1}{|c|}{ Parameter } & Group 1 & Group 2 & $\begin{array}{c}\text { P- } \\
\text { value }\end{array}$ \\
\hline $\begin{array}{l}\text { Average Time needed for } \\
\text { reaching adequate } \\
\text { sedation level (mins) }\end{array}$ & 18.5 & 7.3 & $0.02^{*}$ \\
\hline
\end{tabular}

*:Significant

Table 2: Comparison of clinical parameters

\begin{tabular}{|l|c|c|c|}
\hline Parameter & Group 1 & Group 2 & p-value \\
\hline $\begin{array}{l}\text { Mean infusion } \\
\text { duration (minutes) }\end{array}$ & 53.2 & 55.1 & 0.25 \\
\hline $\begin{array}{l}\text { Mean recovery time } \\
\text { (minutes) }\end{array}$ & 5.42 & 10.45 & $0.01^{*}$ \\
\hline
\end{tabular}

\section{*: Significant}

\section{Discussion}

In the present study, mean recovery time among subjects of group 1 and group 2 was 5.42 and 10.45 minutes respectively. In one of the past studies conducted by Bresil P et al, authors concluded that BIS monitoring for guidance of propofol-remifentanil anaesthesia does not lead to reduction in consumption of anaesthetics, with no reduction of time to extubation in adult and paediatric subjects in comparison to the conventional practice. ${ }^{10}$ Assessment of BIS following propofol induction was done by Arya et al, on the dosage requirement of propofol along with its haemodynamic effects and BIS alterations. A total of 70 patients were assessed by them which were planned to undergo elective surgery under general anaesthesia. They randomly divided the patients into two study groups- group A and group B. Group A comprised of patients who were given (inj.) fentanyl $(2 \mu \mathrm{g} / \mathrm{kg})$, followed 3 min later by inj. propofol at the rate of 30 $\mathrm{mg} / \mathrm{kg} / \mathrm{hr}$ infusion to the point of loss of response to verbal command. Group B comprised of patients that inj. fentanyl $(2 \mu \mathrm{g} / \mathrm{kg})$, followed $3 \mathrm{~min}$ later by inj. propofol at the rate of $30 \mathrm{mg} / \mathrm{kg} / \mathrm{hr}$ infusion. From the results, they concluded that in relation to the induction dose of propofol, no significant difference exists, when assessed clinically or through BIS monitoring. ${ }^{11}$ In another study conducted by Lo YL et al, the authors didn't observe any significant difference in relation to the quantity of patients with hypotensive events. ${ }^{12}$ Banerjee A et al, in one of the previous studies, recognized the impact of caudal epidural block on upkeep need of IV propofol in targeted BIS checked subjects. They initially administered general anaesthesia to children, followed by administration of caudal analgesia and IV analgesia, with the aim of monitoring the propofol infusion intra-surgical requirement. They did this by using BIS monitoring system. Total 82 children within the age group of three years to six years were evaluated by them, who were scheduled to undergo infra-umbilical surgery. They randomly made division of all the patients into two 
broad groups; group A and group B with 41 patients in each group. By analysing the results, the authors came up to conclusion that a decline in the consumption of IV propofol occurred in BIS-monitored paediatric subjects. ${ }^{13}$

In the epidural space, mechanism of action of anaesthetic solution occurs by three prominent ways. First mechanism says that most crucial factor in the arousal from anaesthesia is played by pain factor. From the surgical site, blockage of pain occurs by caudal analgesia. This leads to a decline in the demand of general anesthesia. Second mechanism says that reduction in the anaesthetic requirement occurs by neuraxial block. This occurs by suppression of the movement in reaction to toxic incitement under the level of sensory block. Third mechanism states that brain is directly affected by the rostral movement of the local anesthetic in CSF. ${ }^{14}$ In another study conducted by Agarwal A et al, authors assessed the impact of LA solutions on the balanced anaesthesia components. These components included hypnosis, analgesia, and muscle relaxation. The authors observed that during the phase of anaesthesia maintenance, there is significant decline in the need of vecuronium and fentanyl in the bupivacaine group. By analysis of the results, they concluded that during general anaesthesia administration, reduction in the need of propofol, fentanyl, and vecuronium infusion occurs in subjects in which epidural bupivacaine was administered prior to induction of anesthesia reduces the requirement of propofol, fentanyl, and vecuronium during general anesthesia. $^{15}$ Results from previous studies also established that fact that by performing caudal blockage, there occurs a decline in the requirement of intraoperative inhalation anesthetic like sevoflurane. ${ }^{12-16}$

\section{Conclusion}

From the above results, it can be concluded that BIS monitoring should be done for assessing the level of sedation in regional anaesthesia cases. This results in reduction in the total dose of propofol needed. However; further studies are recommended in future in this field for better exploration of this field of anaesthesia.

\section{Conflict of Interest: None}

\section{References}

1. Singh H, Sakai T, Matsuki A. Movement response to skin incision: Analgesia vs. bispectral index and 95\% spectral edge frequency. Eur J Anaesth. 1999;16:610-614.

2. Kingston S, Mao L, Yang L, Arora A, Fibuch EE, Wang JQ. Propofol inhibits phosphorylation of N-methyl-Daspartate receptor NR1 subunits in neurons. Anesthesiology. 2006;104(4):763-769.

3. Cattano D, Young C, Straiko MM, Olney JW. Subanesthetic doses of propofol induce neuroapoptosis in the infant mouse brain. Anesth and analgesia. 2008;106(6):1712-1714.
4. Clark G, Licker M, Younossian AB, Soccal PM, Frey JG. Titrated sedation with propofol or midazolam for flexible bronchoscopy: a randomised trial. Eur Respir J. 2009;34:1277-1283.

5. Creeley C, Dikranian K, Dissen G, Martin L, Olney J, Brambrink A. Propofol-induced apoptosis of neurones and oligodendrocytes in fetal and neonatal rhesus macaque brain. Br J Anaesth. 2013;110(Suppl 1):i29-38.

6. Anker-Moller E, Spangsberg N, Arendt-Nielsen L, Schultz P, Kristensen MS, Bjerring P. Subhypnotic doses of thiopentone and propofol cause analgesia to experimentally induced acute pain. Br J Anaesth. 1991;66(2):185-188.

7. Stolz D, Kurer G, Meyer A, Chhajed PN, Pflimlin E, et al. Propofol versus combined sedation in flexible bronchoscopy: a randomised non-inferiority trial. Eur Respir J. 2009;34:1024-1030.

8. Rinaldi S, Consales G, DeGaudio AR. State entropy and Bispectral index: Correlation with end tidal sevoflurane concentrations. Minerva Anestesiol. 2007;73:39-48.

9. Rampil IJ, Kim JS, Lenhardt R. Bispectral EEG index during nitrous oxide administration. Anesthesiology. 1998;89:671-677.

10. Bresil P1, Nielsson MS, Malver LP, Kraemer K, Schjørring O, Dethlefsen C, Lambert PH. Impact of bispectral index for monitoring propofol remifentanil anaesthesia. A randomised clinical trial. Acta Anaesthesiol Scand. 2013;57(8):978-987.

11. Arya S, Asthana V, Sharma JP. Clinical vs. bispectral index-guided propofol induction of anaesthesia: A comparative study. Saudi J Anaesth. 2013;7(1):75-79.

12. Lo Y-L, Lin T-Y, Fang Y-F. Feasibility of Bispectral Index-Guided Propofol Infusion for Flexible Bronchoscopy Sedation: A Randomized Controlled Trial. Reddy R, ed. PLoS ONE. 2011;6(11):e27769.

13. Banerjee A, Das B, Mukherjee D, Khanra M. A study of the effect of caudal epidural block on bispectral index targeted propofol requirement in children: A comparative study. J Indian Assoc of Pediatr Surg. 2015;20(2):77-81.

14. Iida R, Iwasaki K, Kato J, Ogawa S. Bispectral index is related to the spread of spinal sensory block in patients with combined spinal and general anaesthesia. $\mathrm{Br} \mathrm{J}$ Anaesth. 2011;106:202-207.

15. Agarwal A1, Pandey R, Dhiraaj S, Singh PK, Raza M, Pandey CK, Gupta D, Choudhury A, Singh U. The effect of epidural bupivacaine on induction and maintenance doses of propofol (evaluated by bispectral index) and maintenance doses of fentanyl and vecuronium. Anesth Analg. 2004;99(6):1684-1688.

16. Doufas AG, Wadhwa A, Shah YM, Lin CM, Haugh GS, Sessler DI. Block-dependent sedation during epidural anaesthesia is associated with delayed brainstem conduction. Br J Anaesth. 2004;93:228-234.

How to cite this article: Singh $\mathrm{R} P$, Arora $\mathrm{M}$. Comparison of dose requirement of propofol with and without bispectral monitoring in patients undergoing spinal epidural anaesthesia: An institutional based study. Indian J Clin Anaesth. 2018;5(4):566-568. 